\title{
FASP médicale et substrat professionnel : le miroir éclaté
}

Jean-Pierre Charpy

\section{(2) OpenEdition}

1 Journals

Édition électronique

URL : http://journals.openedition.org/asp/955

DOI : 10.4000/asp.955

ISBN : 978-2-8218-0412-8

ISSN : 2108-6354

\section{Éditeur}

Groupe d'étude et de recherche en anglais de spécialité

Édition imprimée

Date de publication : 30 mars 2010

Pagination : 49-65

ISSN : 1246-8185

\section{Référence électronique}

Jean-Pierre Charpy, «FASP médicale et substrat professionnel : le miroir éclaté », ASp [En ligne], 57 |

2010, mis en ligne le 01 mars 2013, consulté le 02 novembre 2020. URL : http://

journals.openedition.org/asp/955; DOI : https://doi.org/10.4000/asp.955

Ce document a été généré automatiquement le 2 novembre 2020.

Tous droits réservés 


\title{
FASP médicale et substrat professionnel : le miroir éclaté
}

\author{
Jean-Pierre Charpy
}

\section{Introduction}

1 Voilà dix ans déjà que Michel Petit a codifié le genre de la FASP. Il a donné corps à ce genre littéraire latent, mais non identifié en tant que tel à l'époque. Dans deux articles fondateurs $(1999,2000)$, il a défini les notions de «substrat professionnel », ainsi que d'«adstrat professionnel »ou d'«adstrat spécialisé ». Il a également établi une distinction précieuse entre «milieu professionnel réel» et «milieu professionnel représenté dans la fiction » (2004). D'autres chercheurs, comme Shaeda Isani (2004), ont mis au jour les multiples facettes de la FASP, en étudiant les mutations du genre et la perception par des lecteurs non initiés d'œuvres de fiction spécialisée.

2 Les productions commerciales contemporaines répertoriées à ce jour constituent un bloc, a priori homogène. Cet ensemble est caractérisé par l'appartenance ou l'affiliation des auteurs à un milieu professionnel donné (la justice, la médecine, l'économie, le monde universitaire...), par un contenu narratif et discursif à dominante professionnelle et un recours systématique au suspense et aux rebondissements propres au genre littéraire du thriller. On notera que cette typologie laisse entendre que ces productions sont, pour ainsi dire, le miroir du réel des divers milieux professionnels représentés.

3 En raison de la multiplicité des domaines professionnels qu'elle englobe, la matrice de la FASP a produit des genres particuliers (FASP économique, FASP juridique, FASP médicale, FASP militaire, FASP universitaire...), qui forment des groupes génériques spécialisés. Ces groupes constituent désormais un objet de recherche pertinent dans une perspective diachronique, puisque la publication des romans de la FASP est vivace et perdure depuis plus de trente ans déjà.

4 Dans cette étude nous nous concentrerons exclusivement sur les œuvres de fiction de la FASP médicale, dans la continuité de travaux de recherche entrepris depuis quelques 
années (Charpy 2004a, 2004b, 2005). Jusqu'à ce jour, nous avons fait référence aux « medical thrillers" du genre spécialisé en utilisant l'expression "romans à suspense à dominante médicale » (2004a : 63) puisque leur contenu est principalement, mais non exclusivement, médical. Toutefois, cette appellation même semble être remise en cause par un processus propre à la variation des genres, à savoir une évolution progressive vers une dominante émotionnelle et l'intégration d'adstrats professionnels en provenance de genres spécialisés connexes.

5 Ainsi, les derniers romans de Tess Gerritsen et en particulier un roman récent de Robin Cook(Crisis 2006) illustrent-ils cette évolution par osmose car ils ne répondent plus aux critères strictement normatifs du roman à suspense à dominante médicale. En effet, le réel fictif n'y reflète plus majoritairement le réel professionnel de la communauté médicale.

On peut alors se demander légitimement si ces diverses tendances correspondent à de simples mutations génériques, influant seulement sur la perception optique du genre spécialisé, ou si elles ne remettent pas en cause certains aspects définitoires de la FASP en faisant éclater le miroir tendu par Michel Petit il y a dix ans.

\section{Le miroir plan : typologie des romans à suspense à dominante médicale}

\subsection{La FASP médicale confrontée au miroir du genre : un genre spécialisé de la FASP}

7 L'acronyme générique FASP peut paraître limitatif, car une production fondée sur le substrat professionnel biomédical, comme The Hot Zone (Preston 1994), est définie tantôt comme "a riveting nonfiction thriller", tantôt comme "a fast-paced, gripping medical thriller» (extraits du paratexte éditorial 1995). Toutefois, il est avéré que, pour recevoir l'appellation FASP, les productions du genre doivent émaner ou s'inspirer principalement du réel professionnel d'un (ou de plusieurs) milieu(x) professionnel(s).

8 En ce qui concerne les termes définitoires essentiels du genre, la forte prégnance du substrat ou de l'adstrat professionnel dans les productions de la FASP peut être mesurée à l'aune du statut professionnel, passé ou présent, des auteurs, à leur intégration indirecte au milieu professionnel en tant qu'observateurs, mais surtout à la validation du substrat ou de l'adstrat auprès des praticiens du domaine, garants de la réalité socioprofessionnelle préexistante. Le reste relève du strict champ de la fiction.

Le genre général de la FASP, en tant qu'objet d'étude, est subdivisé en genres particuliers qui constituent des groupes génériques spécialisés. La FASP médicale s'inscrit clairement dans le contexte de la fiction à substrat professionnel. Dans trois articles précédents (Charpy 2004a, 2004b, 2005)' nous avons tenté de dessiner le cœur et les contours de ce domaine tout en repérant ses interconnections avec le milieu professionnel de la médecine. 


\subsection{La FASP médicaleconfrontée au miroir du temps : pertinence d'une étude diachronique}

désormais une cohérence diachronique permettant de repérer les articulations et l'évolution du genre spécialisé. En effet, nous avons suffisamment de recul pour pouvoir en étudier les principales caractéristiques diachroniques, puisque Frank Slaughter, un de ses inspirateurs, a publié ses principaux romans médicaux au milieu du XXe siècle avec Air Surgeon (1943)etBattle Surgeon (1944), et que Michael Crichton ${ }^{1}$, récemment disparu, a contribué à poser les fondations de la FASP médicale, à la fin des années 1960 avec A Case of Need (1968) et The Terminal Man (1972).

11 Il convient de noter que le genre des medical thrillers est fondamentalement d'essence nord-américaine et qu'il s'est développé dans le terreau médical fertile de la NouvelleAngleterre. À la fin des années 1970, Robin Cook (Coma 1977) et Michael Palmer (The Sisterhood 1982) ont ancré ce genre spécialisé dans le paysage éditorial commercial des États-Unis. C'est alors que les romans à suspense à dominante médicale ont constitué un genre populaire à part entière.

12 Certains auteurs, comme le médecin irlandais Paul Carson (Final Duty 2000) et le chercheur écossais Ken McLure (Past Lives 2006), ont exploité la veine de la FASP médicale ou biomédicale en Europe mais, à ce jour, la production de masse de ces romans reste une spécialité nord-américaine. Aux États-Unis quarante ans après leur début, la ferveur des pionniers du genre n'est pas retombée puisque Robin Cook et Michael Palmer continuent de publier régulièrement et que des auteurs reconnus de la deuxième génération comme Tess Gerritsen (Under the Knife 1990, Harvest 1996, The Surgeon 2001) ou un novice comme Michael Esposito (Locked in 2007) ${ }^{2}$ ont repris ou sont en train de reprendre le flambeau de la FASP médicale.

13 Cet empan diachronique respectable devrait nous permettre de jeter un regard plus distancié sur le genre spécialisé et de mieux cerner son évolution. Le substrat professionnel qui étaye les romans à suspense à dominante médicale doit donc être défini et les contours de la FASP médicale doivent être délimités.

\subsection{La FASP médicale confrontée au miroir plan : stéréotypie des romans de la FASP médicale}

14 L'appellation medical thrillers est le résultat d'un croisement générique qui relève de la manipulation génétique. Au chromosome propre du genre littéraire avéré du thriller (intrigue à rebondissements, forte dose de suspense, exacerbation des émotions du lecteur...) les auteurs ont ajouté le gène du substrat professionnel.

Les éditeurs ne se sont pas privés de mettre en relief cette hybridation. Dans une communication faite en $2009^{3}$, nous avons montré que le péritexte éditorial de 27 romans écrits par des auteurs de la FASP médicale (Gary Braver, Michael Crichton, Robin Cook, Tess Gerritsen, Leonard S. Goldberg, Michael Palmer, Leah Ruth Robinson), contribuait à donner une vision stéréotypée du genre.

16 Les occurrences, répertoriées par nos soins, des mots clés présents dans le paratexte éditorial («suspense / suspenseful »(24 occurrences) ${ }^{4}$; «frightening / scary / terrifying »(15) ; « riveting / gripping»(13); «fast-paced »et synonymes (11); «page-turner »et 
synonymes (9) ; « medical thriller » (9)...) montrent que les éditeurs font la part belle au suspense et aux émotions au détriment du contenu purement professionnel.

Cette insistance sur le caractère émotionnel des romans est habilement contrebalancée par le péritexte graphique de la première de couverture qui met en valeur le milieu professionnel (caducée (4 occurrences); patient sur un brancard (4); tracé d'électrocardiogramme (4); seringue (3) ; salle d'opération (3)...).

La synthèse éditoriale s'opère généralement dans le slogan de la première ou de la quatrième de couverture, qui vise à conforter le lien entre expérience professionnelle et fiction. Outre le qualificatif de "master of the medical thriller "dont il est systématiquement affublé (six occurrences), Robin Cook est décrit comme «doctorturned-novelist »dans la quatrième de couverture de son roman Invasion (1997).

19 À l'instar des clichés paratextuels introduits pour des raisons de stratégie éditoriale, l'intégration dans la narration des habitudes socioculturelles, des considérations éthiques, mais surtout du discours et des pratiques de la communauté professionnelle peut laisser penser que la FASP médicale offre une représentation fidèle du réel professionnel et des traits caractéristiques du monde de la médecine.

20 La mise en parallèle diachronique de scènes extraites de divers romans de la FASP médicale permet de mettre en lumière une certaine stéréotypie narrative et discursive. Dans cette scène de Coma située au service des soins intensifs, Robin Cook pose le cadre narratif et discursif stéréotypé du genre.

"Everyone away from the bed!" ordered Bellows. His right thumb made contact and a powerful electric charge spread through Nancy Greenly's chest, arching from one paddle to the other. Her body jerked upward; her arms flopped across her chest with her hands twisting inward. The electronic blip disappeared from the screen, then it returned. It traced a relatively normal pattern.

"She's got a good pulse," said Cartwright.

Reid held up on the external massage. The rate held steady for several minutes. Then a premature ventricular contraction appeared. The rate was again steady for several minutes followed by three premature ventricular contractions in a row.

"V tach," said Shergood confidently. "The heart is still very easily excitable. There has to be something very basic wrong here." (1977 : 92-93)

21 On retrouve, vingt années plus tard, les stéréotypes du genre dans cette scène de Critical Judgement de Michael Palmer,qui se déroule au service des urgences.

"Ready," said the nurse handling the defibrillator console.

"Okay everyone, clear!"

Abby pressed her thumb down on the square plastic button set in the handle of the right-hand paddle. Instantly, there was a muffled pop and an audible, visible spark from two spots where the paddles and skin did not make perfect contact. The man's body - 250 pounds at least - stiffened and arched. His arms snapped upward like whips. Then, just as rapidly, he was still.

"Pump, please," Abby said, checking the monitor screen.

The paramedic, up on a stool for leverage, wiped the contact gel off the man's chest with a towel, set the heel of his hand over the base of the sternum, and resumed his rhythmic compressions. For several seconds there was a slashing up-and-down movement of the tracing on the monitor. But Abby knew from ten years of ER and countless code ninety-nines that the 
pattern was artefact, not related to any effective electrical activity of the heart. (1997: 7-8)

\section{déroule dans une salle d'opération et dans lequel le sentiment d'urgence extrême et le} discours spécialisé sont tout aussi présents.

The blood pressure monitor read 70 over palpable. Her blood pressure had dropped, and the low pressure alarm was now sounding. Her blood pressure was elevated to about 130 . David hung volume expanding fluid to stabilize her pulse rate and blood pressure, but it was not working. He hollered orders to the circulating nurse.

"Get me six units of blood in here. STAT! ' STAT!"

"We never typed and crossed this patient," the circulating nurse yelled back.

"We didn't think we'd need it."

"Get trauma blood! Jesus, it's a fucking emergency!" The anaesthesiologist squeezed a bag of lactated ringer's fluid so it would enter his patient as quickly as possible. “And get me Dr. Hamilton. I need help in here." (2007 : 378)

27
Ces divers extraits montrent que les romans à suspense à dominante médicale cités ci-
dessus reposent sur une base, certes formulaïque, mais facilement reconnaissable par 
les professionnels du monde de la médecine. Il semble bien y avoir adéquation entre le réel professionnel et la représentation qui en est faite dans l'œuvre de fiction, qui renvoie une image globalement fidèle des pratiques, du discours et des habitudes professionnelles et socioculturelles de la communauté médicale.

\section{De l'autre côté du miroir : l'image déformée de la FASP médicale}

\subsection{Harmonie apparente entre réalité professionnelle et fiction}

Dans l'avertissement de l'auteur à propos de Seizure, Robin Cook aborde la question des relations entre le réel et la fiction dans ses romans: "I think of my novels as " faction ", a coined word meaning that the facts and fiction are so mixed that the dividing line between the two is often hard to discern." (2003: Author's note)

Á première vue, ce néologisme semble séduisant tant il est vrai que Robin Cook et d'autres auteurs, comme Michael Palmer, s'appuient sur la réalité médicale ou sur des considérations d'éthique médicale qui parcourent la société américaine pour tisser leur fil narratif et discursif.

Ces écrivains utilisent certains aspects du réel professionnel qu'ils transforment par l'écriture en réel fictif à des fins souvent pédagogiques. Certes, il s'agit de divertir le lecteur, mais aussi de l'informer et de l'éclairer. Ces objectifs apparaissent clairement dans cette note de Michael Palmer adressée aux lecteurs à la fin de The Fifth Vial.

My goal in writing suspense is first and foremost to entertain my readers and to transport them, however transiently, from the stresses and cares of their lives to the highly stylized world of the novel. My secondary goals are to inform and to present, without resolution, issues of social and ethical importance. (2007: 501)

31 Robin Cook est coutumier de ces avertissements aux lecteurs qui lui permettent d'affirmer le lien étroit entre réalité et fiction. C'est le cas dans son premier roman à suspense à dominante médicale, Coma: "This novel was conceived as an entertainment, but it is not science fiction. Its implications are scary because they are possible, perhaps even probable" (1978: 329).

Dans les romans de Robin Cook, le réel fictif reflète souvent les préoccupations sociales ou éthiques réelles de la communauté médicale, qu'il s'agisse de trafic d'organes (Coma), de manipulations génétiques (Chromosome 6), de médecine "à deux vitesses " (Crisis) ou de bio-terrorisme comme dans cet extrait de Vector.

Yet the medical profession's responsibility with regard to bioterrorism goes beyond detecting an episode and treating its victims. The medical profession has an ethical duty to continue to institutionalize the opprobrium currently associated with use of bioweapons. $(2000: 389)$

Toutefois, cette image harmonieuse qui est mise en avant dans certains romans, perd de sa clarté dans plusieurs medical thrillers publiés récemment. 


\subsection{Tensions entre réalité professionnelle et fiction}

Dans un roman dit à dominante professionnelle, le lecteur, qu'il soit profane ou membre de la communauté professionnelle, s'attend à une certaine adéquation entre réel professionnel et réel fictif. Bien que le discours spécialisé et le savoir technique présents dans le roman n'offrent qu'une image virtuelle, ni totalement fidèle ni complètement neutre en raison du vécu et de l'imaginaire de l'auteur, il n'en reste pas moins que cette image devrait être le reflet fictif de la réalité d'une communauté professionnelle donnée.

Or, il se trouve que dans de nombreux romans, pourtant identifiés dans le paratexte éditorial comme medical thrillers, l'image du réel professionnel dans l'œuvre de fiction est réduite à la portion congrue.

Ainsi, dans Past Lives (2006), Ken McClure ne consacre-t-il que quelques pages au substrat professionnel médical alors que l'intérêt du roman repose essentiellement sur une intrigue haletante, qui n'est pas sans rappeler celle du Da Vinci Code de Dan Brown (2003). L'onde de choc émotionnelle balaye sur son passage la représentation du réel professionnel et le réduit à une peau de chagrin.

De la même manière, The First Patient (Palmer 2008) est un roman de 484 pages dont une quinzaine de pages seulement comportent des éléments significatifs de substrat professionnel médical. En outre, le paratexte éditorial révèle un glissement du professionnel vers l'émotionnel. Dans la première de couverture, contrairement à l'habitude, le livre est présenté comme " a novel», un simple roman, et non comme un "medical thriller».

Ce glissement vers les aspects définitoires du genre du thrillerest confirmé par le graphisme même de la première de couverture - une vue stylisée de la Maison Blanche - et par le commentaire de bas de page d'un lecteur quelque peu inattendu : "An exciting thriller that is full of surprises... captures the intense atmosphere of the White House. PRESIDENT BILL CLINTON".

Dans ses romans les plus récents, Tess Gerritsen a délaissé le terrain de l'intrigue criminelle se déroulant dans le milieu médical, qui était sa marque de fabrique jusqu'à la fin des années 90, pour écrire des romans dont l'objectif principal est d'exacerber les émotions des lecteurs avides de mystère et de suspense.

Dans un entretien électronique de mars 2009 avec l'auteur de cet article, l'écrivain définit son lectorat-cible : "I write for an audience much like myself - and I love scary, hightension books that emphasize science".

41 Il convient de noter que Tess Gerritsen ne privilégie pas des caractéristiques professionnelles spécifiques, mais s'en tient à des considérations scientifiques générales; en revanche, elle présente comme traits définitoires de ses romans les éléments de suspense et un certain goût de l'épouvante qu'elle partage avec ses lecteurs.

42 Au sujet du genre spécialisé de la FASP médicale, qui nous intéresse ici au premier chef, elle ajoute :

Si l'on s'en tient à cette définition, la production actuelle de medical thrillers reflétant le réel professionnel et les considérations éthiques de la communauté médicale serait limitée aujourd'hui à un nombre restreint de romans écrits par Robin Cook et Michael Palmer ou à des productions récentes, majoritairement consacrées au monde de la 
médecine spécialisée, en l'occurrence la radiologie, comme Locked In de Michael Esposito.

\section{Le miroir éclaté : évolution(s) du genre spécialisé}

\subsection{Une nouvelle facette du genre spécialisé : les emprunts à un genre connexe} (2004:28). discursif :

Bien qu'étant fréquemment classés par les éditeurs comme des genres spécialisés distincts, les medical thrillers et les forensic thrillers sont à l'interface de la science, de la médecine et de la justice. Il n'est donc pas étonnant de retrouver des influences conjointes chez certains auteurs de la FASP médicale.

Comme nous l'avons indiqué précédemment, Tess Gerritsen est l'un des auteurs emblématiques de la FASP médicale. Elle a construit sa réputation dans ce domaine avec Harvest (1996), mais il est intéressant de noter que Under the Knife (1990), répertorié à l'époque à tort comme un simple "romantic thriller ", présente déjà de nombreux traits stéréotypiques du roman à suspense à dominante médicale.

C'est dans The Apprentice (2002) que l'auteur introduit de façon suivie le personnage du Dr Maura Isles, une femme médecin légiste qui est amenée à travailler avec le détective Jane Rizzoli. Elle avait reçu un surnom évocateur: "The Queen of the Dead was what the homicide unit had dubbed her a year ago, when she'd first joined the Commonwealth of Massachusetts medical examiner's office" (2002:39).

Un an plus tard, le roman The Sinner (2003)présente des traits qui le rattachent à la fois à la catégorie des medical thrillers et à ce que Shaeda Isani appelle "The Panachée FASP "

Tess Gerritsen y met sa connaissance personnelle de la médecine au service du substrat professionnel médical et elle y ajoute un volet original avec l'utilisation d'adstrat professionnel lié à la présence du médecin légiste Maura Isles, qui partage désormais le devant de la scène aux côtés de l'héroïne des romans précédents, le détective Jane Rizzoli : "Together, Isles and homicide detective Jane Rizzoli uncover an ancient horror that connects these terrible slaughters" (2003 : quatrième de couverture).

Les années suivantes, Body Double (2004) et The Mephisto Club (2006)confirment ces emprunts et l'empreinte de la médecine légale dans la trame narrative et le tissu

Kathy Reichs (Death du Jour 1999), l'une des spécialiste du genre du forensic thriller, met pleinement à profit sa parfaite connaissance du discours et des conventions de l'anthropologie médico-légale, qu'elle exploite de l'intérieur puisqu'elle a une expertise mondialement reconnue dans cette pratique professionnelle.

De la même manière, Tess Gerritsen intègre depuis quelques années, de l'extérieur, des éléments de médecine légaleen faisant appel à l'expertise de spécialistes du domaine, comme elle le reconnaît dans The Sinner : "My warmest thanks to: [...] Margaret Greenwald, M.D., for allowing me a look into the medical examiner's world" (2004 : Acknowledgements).

52 Cette hybridation accrue d'un genre déjà hybride n'est pas l'apanage de Tess Gerritsen, car elle n'est pas le seul auteur de la FASP médicale à combiner des scènes de médecine traditionnelle et des scènes de médecine légale. Le personnage central des romans de 
Leonard S. Goldberg, le docteur Joanna Blalock, est une pathologiste spécialisée dans la médecine légale. De même, l'un des personnages récurrents de Robin Cook, Jack Stapleton, est médecin légiste de profession.

\subsection{Un miroir aux multiples facettes :l'exemple de Crisis}

53 En 2006, le père fondateur du roman à suspense à dominante médicale, Robin Cook, publie Crisis, une forme hybride protéiforme reposant sur l'osmose de trois genres spécialisés existants. En effet, on y trouve l'adstrat lexical spécialisé des romans de la FASP juridique aux côtés de l'adstrat spécialisé de la médecine légale et du substrat professionnel de la médecine.

À la lecture du texte de présentation de Crisis on pourrait même avancer que ce roman de la FASP est le premier "forensic-medical-legal thriller ». Les éléments du paratexte éditorial (mis en italiques par nos soins) reflètent cette évolution :

A partner in an exclusive concierge medical practice, Dr. Craig Bowman, has reached an impressive level of success. But this idyllic situation comes to a grinding halt when he's served with a summons for medical negligence - and things get much, much worse. Enter Dr. Jack Stapleton, a medical examiner in New York City and Craig's brother-in-law, who agrees to travel to Boston to offer his forensic services and expert-witness experience to Craig's beleaguered defense attorney. But when Jack's irreverent suggestion to exhume the corpse, and thereby disprove the alleged malpractice, is taken seriously, he opens a Pandora's box. As Craig's life and career are put on the line, Jack is on the verge of making a most unwelcome discovery, of tremendous legal and medical significance - and there are people who will do anything to keep him from learning the truth. $(2006: \mathrm{i})$

La lecture du paratexte auctorial est également édifiante. En exergue du roman, dans la partie "Acknowledgements» (remerciements), l'auteur reconnaît sa dette envers deux médecins légistes et quatre juristes, grâce auxquels il a pu s'approprier l'adstrat professionnel indispensable à la cohérence narrative et discursive de son roman hybride :

As usual in writing my fact-based novels, I've had to rely on friends and acquaintances to answer my innumerable pesky questions. It was especially important for Crisis, since the story line bridges medicine and law. (2006 : vii)

L'intrigue de Crisis repose sur la fusion aboutie d'éléments de substrat professionnel médical classique et d'éléments d'adstrat professionnel légal et médico-légal, ainsi que sur le maillage de scènes reflétant les caractéristiques des trois milieux professionnels représentés.

Le docteur Craig Bowman, le protagoniste impliqué dans le procès pour faute professionnelle, est à l'origine de l'essentiel du substrat professionnel médical présent dans le roman. Dans cette scène du prologue, on retrouve les stéréotypes de la FASP médicale décrits plus haut (voir supra, 1.3.).

\footnotetext{
"Is she going to be all right?" Leona asked in a whisper.

"Who the hell knows," Craig answered." She's cyanotic, for Christ's sake."

"What's cyanotic?

“There's not enough oxygen in her blood. I don't know if it's because her heart isn't pumping enough or she's not breathing enough. It's one or the
} 
other or both."

Craig concentrated on the ECG machine as it spewed out a tracing. There were only little blips, widely spaced. Craig tore off the output strip and took a quick, closer glance at it before jamming it into his jacket pocket. He then snapped the leads off Patience's extremities. (2006:17)

Des éléments d'adstrat professionnel médico-légal émaillent également la trame narrative et discursive du roman. Cette combinaison n'est pas nouvelle chez Robin Cook car Jack Stapleton, le médecin légiste qui va contribuer professionnellement au dénouement, était déjà l'un des personnages principaux de Chromosome 6 (1997). Le passage qui suit illustre cette expertise mise au service de l'intrigue :

By merely palpating the surface of the lungs, Jack's experienced hands perceived an abnormal resistance. The tissue was firmer than usual, which was consistent with the organ's weight being higher than normal. With a knife that looked like a garden-variety butcher-knife, Jack made multiple slices into the lungs. Again, there was the suggestion of more resistance than he would expect. Lifting the lung, he examined the cut surfaces, which reflected the organ's consistency. The lung appeared denser than normal, and he was confident that the microscope would show fibrosis. The question was ... why were the lungs fibrotic? $(2006: 65)$

Néanmoins, l'élément le plus inattendu du roman et, de ce fait, le plus novateur est l'intégration filée d'adstrat professionnel juridique. Cette scène de tribunal en est une parfaite illustration :

“Objection!" Randolph called out, leaping to his feet.

"And move to strike and request the court to admonish counsel. The tribunal's findings are not admissible: Beeler versus Downey, Massachusetts Supreme Judicial Court."

"Sustained!" Judge Davidson snapped. "Defense counsel is correct, Mr. Fassano."

"I'm sorry, Your Honor," Tony said. He stepped over to the plaintiff's table and took a paper offered by Ms. Relf. "I have here a copy of Massachusetts Laws, Chapter two thirty-one, section sixty B, saying the panel's findings and testimony before the panel are admissible." (2006:103)

Des scènes au tribunal ou autour du tribunal se succèdent dans Crisis. En réalité, son intrigue est étroitement liée au milieu professionnel de la justice puisque le sort du docteur Bowman dépend avant tout de l'issue du procès auquel il est confronté pour faute professionnelle.

61 Le lexique spécialisé, parfois suranné, de la justice (« the voir dire proceeding » (p. 77), a « tortfeaser » (p. 79), «veniremen »(p. 81), «prima facie case» (p. 96)...) contraste avec la terminologie médicale qui caractérise habituellement les medical thrillers.

Force est de constater, au travers de ces quelques illustrations tirées de Crisis, que le reflet dominant n'est plus exclusivement celui du substrat professionnel médical et que de nombreux éléments d'adstrat professionnel provenant de la médecine légaleou du milieu judiciaire sont entrelacés dans le tissu narratif et discursif du roman. 


\section{Conclusion}

de quelques variations professionnelles. Comme les genres spécialisés qu'elle regroupe en son sein, la FASP se transforme et évolue vers des formes romanesques que nous préférons qualifier de «thrillers à valeur professionnelle ajoutée ».

70 Après avoir épuisé les thèmes centraux du genre spécialisé, les écrivains de la FASP médicale se livrent à des variations génériques pour entretenir l'intérêt de leurs lecteurs habituels. En outre, étant donné le contexte actuel où la concurrence éditoriale est féroce, ils s'efforcent d'attirer de nouveaux lecteurs.

71 Sans délaisser totalement les critères fondamentaux du roman à suspense à dominante médicale, en particulier ses aspects éthiques et socioculturels, les auteurs des textes fondateurs originaux absorbent progressivement des éléments constitutifs de genres à forte résonance sociétale (les affaires judiciaires du legal thriller) ou de genres spécialisés connexes à fort impact médiatique (le forensic thriller). 


\section{BIBLIOGRAPHIE}

\section{Corpus}

Carson, Paul. 2001 [2000]. Final Duty. Londres :Arrow Books.

Connelly, Michael. 2006 [2005]. The Lincoln Lawyer. New York :Grand Central Publishing.

Connelly, Michael. 2009 [2008]. The Brass Verdict. Londres :Orion Books.

Cook, Robin. 1978 [1977]. Coma. Londres : Pan Books.

Cook, Robin. 1997. Invasion. New York : Berkley Books.

Cook, Robin. 1998 [1997]. Chromosome 6. New York : Berkley Books.

Cook, Robin. 2000 [1999]. Vector. New York : Berkley Fiction.

Cook, Robin. 2003. Seizure. Londres : Pan Books.

Cook, Robin. 2006. Crisis. New York : Berkley Books.

Crichton, Michael (publié sous le nom de Jeffery Hudson). 1969 (1968). A Case of Need. New York :

Signet.

Crichton, Michael. 2002 [1972]. The Terminal Man. New York : Avon Books.

Esposito, Michael. 2007. Locked in. Dallas, TX : Durban House.

Gerritsen, Tess. 2004 [1990]. Under the Knife. Richmond, VA : Mira Books.

Gerritsen, Tess. 1997 [1996]. Harvest. New York : Pocket Star Books.

Gerritsen, Tess. 2002 [2001]. The Surgeon. New York :Ballantine Books.

Gerritsen, Tess. 2004 [2003]. The Sinner. New York :Ballantine Books.

Gerritsen, Tess. 2007 [2006]. The Mephisto Club. New York : Ballantine Books.

McLure, Ken. 2007 [2006]. Past Lives. Londres : Allison \& Busby Limited.

Palmer, Michael. 1995 [1982]. The Sisterhood. New York : Bantam Books.

Palmer, Michael. 1997 [1996]. Critical Judgement. Londres : Arrow Books.

Palmer, Michael. 2007. The Fifth Vial. New York : St. Martin's Paperbacks.

Preston, Richard. 1995 [1994]. The Hot Zone. New York : Anchor Book.

Reichs, Kathy. 1999. Death du Jour. New York : Pocket Books.

Slaughter, Frank. 1943. Air Surgeon. Londres : Hutchinson.

Slaughter, Frank. 1944. Battle Surgeon. Londres : Hutchinson.

Références

Charpy, Jean-Pierre. 2004a. « Le roman à suspense à dominante médicale : typologie d'un sousgroupe générique ». InPetit, Michel et Shaeda Isani (dir.), Aspects de la fiction à substrat professionnel. Collection Travaux EA 2025. Bordeaux : Université Bordeaux 2, 63-80.

Charpy, Jean-Pierre. 2004b. « Milieux professionnels et FASP médicale : de l'autre côté du miroir ». ASp 45-46, 61-79. 
Charpy, Jean-Pierre. 2005. « La FASP médicale et ses marges : textes de référence, prototextes et textes périphériques ». ASp 47-48, 83-101.

Diaz-Santos, Gilberto. 2000. « Technothrillers and English for science and technology ». English for Specific Purposes 19, 221-236.

Genette, Gérard. 1987. Seuils. Paris : Seuil.

Isani, Shaeda. 2004. «The FASP and the genres within the genre ». InPetit, Michel et Shaeda Isani (dir.), Aspects de la fiction à substrat professionnel. Collection Travaux EA 2025. Bordeaux : Université Bordeaux 2, 25-36.

Isani, Shaeda. 2009. « Specialised fictional narrative and lay readership: Bridging the accessibility gap ». ASp 56, 45-65.

Petit, Michel. 1999. « La fiction à substrat professionnel : une autre voie d'accès à l'anglais de spécialité ». ASp 23-26, 57-81.

Petit, Michel. 2000. « Le paratexte dans la fiction à substrat professionnel », Bulletin de la société de stylistique anglaise 21« Texte et paratexte », Actes du Colloque de Nanterre 4-5 juin 1999, 173-195.

Petit, Michel. 2004. «Quelques réflexions sur la fiction à substrat professionnel : du général au particulier ", InPetit, Michel et Shaeda Isani (dir.), Aspects de la fiction à substrat professionnel. Collection Travaux EA 2025. Bordeaux : Université Bordeaux 2, 3-23.

Schaeffer, Jean-Marie. 1989. Qu'est-ce qu'un genre littéraire? Paris : Seuil.

\section{ANNEXES}

Occurrences d'un mot clé dans le paratexte éditorial

\begin{tabular}{|c|c|c|c|c|}
\hline $\begin{array}{c}\text { Mots clés } \\
\text { (nombre } \\
\text { d'occurrences) }\end{array}$ & Auteur & $\begin{array}{l}\text { Titre du } \\
\text { roman }\end{array}$ & Page & Contexte \\
\hline \multirow{14}{*}{$\begin{array}{l}\text { suspense / } \\
\text { suspenseful (24) }\end{array}$} & $\begin{array}{c}\text { M. } \\
\text { Crichton }\end{array}$ & $\begin{array}{l}\text { A Case of } \\
\text { Need }\end{array}$ & $\begin{array}{l}\text { D. } 1 \\
\text { quatrième } \\
\text { de } \\
\text { couverture }\end{array}$ & $\begin{array}{l}\text { "breathtaking } \\
\text { suspense" } \\
\text { "The classic novel of } \\
\text { suspense" }\end{array}$ \\
\hline & \multirow[t]{4}{*}{ R. Cook } & Fever & $\begin{array}{l}\text { quatrieme } \\
\text { de } \\
\text { couverture }\end{array}$ & $\begin{array}{l}\text { "riveting } \\
\text { suspense" } \\
\text { "masterful } \\
\text { suspense" }\end{array}$ \\
\hline & & Vital Signs & 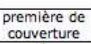 & $\begin{array}{l}\text { "Constant } \\
\text { suspense!" }\end{array}$ \\
\hline & & Terminal & & $\begin{array}{l}\text { ".shock and } \\
\text { suspense" }\end{array}$ \\
\hline & & $\begin{array}{c}\text { Chromosome } \\
6\end{array}$ & $\begin{array}{l}\text { quatrième } \\
\text { de } \\
\text { couverture }\end{array}$ & $\begin{array}{l}\text { suspense master } \\
\text { Robin Cook" }\end{array}$ \\
\hline & \multirow[t]{5}{*}{$\begin{array}{c}\cdot \\
\text { Gerritsen } \\
\end{array}$} & Harvest & 1 & $\begin{array}{l}\text { "the tale of medical } \\
\text { suspense" } \\
\text { "suspense as sharp } \\
\text { as a scalpel's edge" }\end{array}$ \\
\hline & & Life Support & $\begin{array}{c}\text { première de } \\
\text { couverture } \\
1\end{array}$ & $\begin{array}{l}\text { a novel of medical } \\
\text { suspense" } \\
\text { "Another } \\
\text { suspenseful } \\
\text { read " }\end{array}$ \\
\hline & & Gravity & $\begin{array}{l}\text { première de } \\
\text { couverture } \\
1\end{array}$ & $\begin{array}{l}\text { "a novel of medical } \\
\text { suspense" } \\
\text { "medical } \\
\text { suspense" }\end{array}$ \\
\hline & & $\begin{array}{l}\text { The } \\
\text { Apprentice }\end{array}$ & $\begin{array}{l}\text { quatrième } \\
\text { de } \\
\text { couverture }\end{array}$ & $\begin{array}{l}\text { "The suspense is } \\
\text { palpable." }\end{array}$ \\
\hline & & Body Double & $\begin{array}{c}\text { première de } \\
\text { couverture } \\
1 \\
1\end{array}$ & $\begin{array}{l}\text { "chilling suspense" } \\
\text { "Richly suspenseful } \\
\text { and provocative.." } \\
\text { "Gerritsen does a } \\
\text { masterful job of } \\
\text { ratcheting up the } \\
\text { suspense." }\end{array}$ \\
\hline & \multirow[t]{3}{*}{$\begin{array}{l}\text { M. } \\
\text { Palmer }\end{array}$} & $\begin{array}{l}\text { The } \\
\text { Sistertood }\end{array}$ & $\begin{array}{l}\text { première de } \\
\text { couverture }\end{array}$ & $\begin{array}{l}\text { "A suspenseful } \\
\text { paget tumer..." }\end{array}$ \\
\hline & & The Patient & 1 & $\begin{array}{l}\text { "Twisting. } \\
\text { suspenseful." } \\
\text { "Suspense is } \\
\text { maintained } \\
\text { throughout.." }\end{array}$ \\
\hline & & Fatal & 1 & $\begin{array}{l}\text { "Michael Palmer [is] } \\
\text { a master of medical } \\
\text { suspense..." }\end{array}$ \\
\hline & $\begin{array}{l}\text { L. R. } \\
\text { Robinson }\end{array}$ & Blood Run & i & $\begin{array}{l}\text { "[a] medical } \\
\text { suspense novel" } \\
\text { "The medical } \\
\text { suspense.." } \\
\text { "a suspense- } \\
\text { packed, hard-edged } \\
\text { tale" }\end{array}$ \\
\hline
\end{tabular}




\section{NOTES}

1. Il convient toutefois de noter que Crichton s'est rapidement tourné vers le genre spécialisé du «technothriller».

2. Il est intéressant de noter dès à présent que des éléments d'adstrat juridique sont intégrés à l'intrigue de Under the Knife et de Locked in.

3. "FASP médicale et droit de généricité ", communication donnée lors des journées d'étude ILCEA/GREMUTS «La FASP : dix ans après », organisées par l'Université Stendhal à Grenoble (8-9 juin 2009).

4. Voir annexe.

5. «Premature ventricular contractions».

6. Abréviation du mot latin "statim » (immédiatement), fréquemment utilisée dans les hôpitaux américains dans les situations d'urgence.

\section{RÉSUMÉS}

L'étude des medical thrillers est un domaine de recherche encore jeune dans le contexte de la fiction à substrat professionnel. Ces romans reflètent généralement les pratiques, le discours et les habitudes socioculturelles de la communauté médicale, mais la lecture de romans récents semble indiquer que la représentation du réel professionnel laisse progressivement place au miroir aux alouettes du genre connexe du thriller. Le miroir nous renvoie également une image du réel professionnel plus diversifiée, car les auteurs s'inspirent désormais du substrat spécialisé emprunté aux forensic thrillers et aux legal thrillers. Cette évolution par osmose met en relief le caractère hybride du genre. Au-delà des stéréotypes paratextuels, narratifs et discursifs, l'auteur de l'article s'efforce de montrer, au travers d'extraits récents, la distorsion induite par le tiraillement entre réel professionnel et réel fictif au sein de ces romans. S'il reflète certaines préoccupations du milieu médical, le genre spécialisé néglige souvent sa réalité professionnelle, ce qui remet en cause la notion même de fiction à substrat médical.

The study of medical thrillers is a budding field of research within the genre of professionallybased fiction. These novels usually reflect the practices, the discourse and the socio-cultural ways of the medical community. However, when reading the more recent novels, one has the feeling that the representation of professional reality is gradually giving way to the lure of the closely related thriller genre. In the same way, the image reflected is that of a more diversified professional reality, as the authors now draw their inspiration from professionally-based material borrowed from forensic and legal thrillers. This osmosis-induced evolution highlights the hybrid nature of the genre. The aim of the author's article is to go beyond paratextual, narrative and discursive stereotypes and, through extracts taken from recent novels, to pinpoint distortions induced by the constant tug-of-war between professional reality and reality as portrayed in medical thrillers. Although the specialised genre reflects some of the preoccupations of the medical profession, it often overlooks its professional reality, which questions the very notion of medically-based fiction. 
INDEX

Mots-clés : FASP, FASP médicale, genre hybride, réel fictif, réel professionnel

Keywords : fictional reality, hybrid genre, medically-based fiction, professional reality, professionally-based fiction

\section{AUTEUR \\ JEAN-PIERRE CHARPY}

Jean-Pierre Charpyest maître de conférences à la Faculté de Médecine de Dijon de l'Université de Bourgogne. Il est le co-auteur de La communication orale scientifique en anglais (Ellipses, 2002 et Edulang, 2003), de L'anglais médical à la faculté (Ellipses, 2008) et de L'article de recherche scientifique en anglais (Ellipses, à paraître en 2010). Ses domaines de recherche portent sur la FASP médicale, le discours scientifique formel et l'étude diachronique des dictionnaires médicaux. jeanpierre.charpy@u-bourgogne.fr 